\title{
Análise estatística dos impactos gerados pelo custo amazônico nas compras públicas de uma instituição de ensino
}

\section{Statistical analysis of the impacts generated by the amazon cost on public purchases of an educational intitution}

\author{
Maricélia Alves Soares ${ }^{1 *}$, Marcelo José Raiol Souza ${ }^{2}$, Edilson Marques Magalhães ${ }^{1}$, Bruno \\ Marques Viegas ${ }^{1}$
}

\begin{abstract}
RESUMO
Este trabalho teve como objetivo analisar os impactos gerados pelo custo amazônico em compras públicas em um campus do Instituto Federal de Educação, Ciência e Tecnologia do Amazonas (IFAM). A investigação teve finalidade exploratória e descritiva, estruturada pelo método de estudo de caso com abordagem quali-quantitativa. Na fase qualitativa, avaliou-se os fatores externos no processo de compras que afetam o desempenho das ações institucionais do IFAM. Nessa fase, os dados foram obtidos através de pesquisa documental, aplicação de questionário aberto aos servidores ligados ao setor de compras e entrevista semiestruturada com o diretor da unidade pesquisada. Os dados quantitativos foram obtidos no Portal de Compras Governamental - COMPRASNET e avaliados com estatística descritiva para análise dos custos das compras realizadas por um campus da capital e um interiorano do IFAM. Os resultados da análise indicaram, principalmente, que o fator logístico é o principal responsável pelas diferenças constatadas nos preços entre as unidades presentes na cidade de Manaus e São Gabriel da Cachoeira, onde nesta última apresenta certa precariedade no processo de aquisição de materiais.
\end{abstract}

Palavras-chave: Custo; Análise estatística; Logística.

\section{ABSTRACT}

This work aimed to analyze the impacts generated by the Amazonian cost in public purchases on a campus of the Federal Institute of Education, Science and Technology of Amazonas (IFAM). The investigation had an exploratory and descriptive purpose, structured by the case study method with a quali-quantitative approach. In the qualitative phase, the external factors in the procurement process that affect the performance of IFAM's institutional actions were evaluated. At this stage, data were obtained through documentary research, application of an open questionnaire to servers linked to the purchasing sector and a semi-structured interview with the director of the researched unit. Quantitative data were obtained from the Government Procurement Portal - COMPRASNET and evaluated with descriptive statistics to analyze the costs of purchases carried out by a campus in the capital and an IFAM inland. The results of the analysis indicated, mainly, that the logistic factor is the main responsible for the differences found in the prices

\footnotetext{
${ }^{1}$ Programa de Pós-graduação em Engenharia de Processos, Universidade Federal do Pará, CEP: 66075-110, Belém-Pará, Brasil.

*E-mail: maricelia.peres@gmail.com

${ }^{2}$ Programa de Pós-graduação em Tecnologia, Recursos Naturais e Sustentabilidade na

Amazônia, Universidade do Estado do Pará, CEP: 66095-015, Belém-Pará, Brasil.
} 
between the units present in the city of Manaus and São Gabriel da Cachoeira, where the latter presents a certain precariousness in the process of purchasing materials.

Keywords: Cost; Statistical analysis; Logistics.

\section{INTRODUÇÃO}

Para manter suas atividades fins (ensino, pesquisa e extensão), o Instituto Federal de Educação, Ciência e Tecnologia do Amazonas (IFAM), necessita de uma logística operacional que atenda tanto a capital, quanto o interior do estado. Nesse aspecto, a gestão de compras assume grande relevância para aquisição dos materiais e contratação dos serviços necessários para o alcance dos objetivos da instituição. O IFAM é atualmente constituído por 17 campi, sendo três na capital e 14 no interior do estado, sendo dirigido por um reitor e um diretor geral em cada campus, o qual possui seu próprio organograma de organização administrativa e proposta orçamentária definida pela lei orçamentária anual (IFAM, 2018).

Designa-se como Amazônia Legal ou região Amazônica a área composta pelos estados da região norte acrescidos de parte dos estados do Mato Grosso e Maranhão. Nessa região, os dois maiores estados em área territorial, Amazonas e Pará, impõem desafios devido às grandes distâncias e tempo de viagem. A grande área territorial e a vasta malha hidroviária, sujeita a sazonalidade hídrica, fazem com que as cidades amazônicas dependam basicamente dos rios como indutor da economia local e estadual (SILVA et al., 2017).

Souza e Zanetti (2016) mencionam o reconhecimento pelo ministério da cultura do custo amazônico em 2010, assegurando dotação específica e diferenciada para os estados da Amazônia Legal em projetos e programas educacionais. Diferentemente de outras instituições federais de ensino existentes em outras regiões do Brasil, os campi do IFAM localizados no interior do estado enfrentam problemas de mobilidade e logística, determinados pelas características fisiográficas da região Amazônica, que elevam custos e criam dificuldades para compras e fornecimento de suprimentos para execução das atividades de ensino, pesquisa, extensão e administração.

Acredita-se haver mais dispêndio de recursos públicos nas compras e manutenção

dessas instituições do que em outras regiões brasileiras. Esse gasto adicional e desfavorável, aliada à vasta legislação existente para execução dos processos de 
contratações e compras, compromete a qualidade e eficiência do atendimento às finalidades constitucionais de formação educacional cidadã, cultural e técnico-científica da população atendida pelo instituto federal. Os campi interioranos do IFAM, localizados distantes da capital, são objetos de uma interferência direta da localidade e de logística, o qual pode ser especificado como custo amazônico.

Neste sentido, esta pesquisa busca analisar qualitativamente e quantitativamente o impacto dos custos adicionais nos processos de compras de um campus do IFAM, sujeito às adversidades logísticas de transporte, visando contribuir no aperfeiçoamento dos gastos com bens e serviços realizados pelo setor público.

\section{MATERIAL E MÉTODOS}

Nesta etapa do estudo será apresentado a metodologia utilizada para alcançar os objetivos desta pesquisa, que foi o de analisar o impacto do custo amazônico em compras públicas em um campus do Instituto Federal de Educação do Amazonas. A sequência das etapas utilizadas na pesquisa é apresentada na Figura 1.

Figura 1 - Fluxograma de investigação

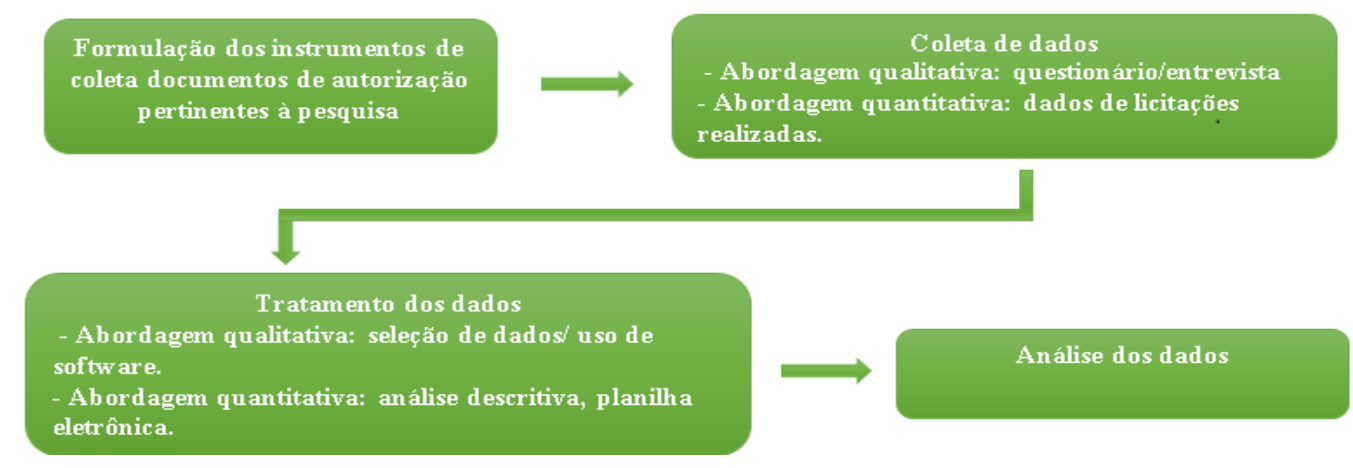

Conforme verifica-se na Figura 1, iniciou-se o trabalho de revisão da literatura para construção do modelo de análise a ser aplicado na investigação. Os instrumentos de coleta de dados qualitativos foram formulados após a aprovação do Comitê de Ética Plataforma Brasil (Parecer $n^{\circ}$ 4.273.041), como questionário aberto e o roteiro da entrevista semiestruturada. Nessa fase, foram solicitadas todas as autorizações legais para realização da pesquisa.

Os instrumentos qualitativos foram aplicados aos servidores ligados às compras e a entrevista semiestruturada foi realizada com o diretor do campus pesquisado. Os dados 
quantitativos relativos às compras realizadas pelos campi da capital e do interior, no período de 2018 a 2020, foram obtidos no Portal de Compras Governamental COMPRASNET. Na fase quantitativa, os dados foram analisados com estatística descritiva para análise matemática dos gastos dos campi em relação à variável de localização.

Pela escassez de estudos e descrição do impacto do custo amazônico em compras públicas no interior do estado do Amazonas, a pesquisa terá finalidade exploratória e descritiva, estruturada pelo método de estudo de caso. Vergara (2016) considera que a “pesquisa exploratória é realizada em área na qual há pouco conhecimento acumulado e sistematizado" e segundo a autora, a pesquisa descritiva "expõe características de determinada população ou de determinado fenômeno".

Define-se o termo custo amazônico como todo gasto adicional advindo das dificuldades estruturais e naturais que afetam o deslocamento, transporte, comunicação e logística de pessoas e mercadorias na região amazônica. $\mathrm{O}$ custo amazônico torna-se fator relevante no aumento do custo de vida nos municípios da região amazônica (COMISSÃO DE DESENVOLVIMENTO URBANO, 2015).

Após trâmites legais, o IFAM recebe seu orçamento geral, que é distribuído aos seus campi, obedecendo a parâmetros pré-estabelecidos. Os campi do IFAM possuem proposta orçamentária anual, exceto no que diz respeito a pessoal, encargos sociais e benefícios aos servidores. O orçamento deverá ser executado em conformidade com o plano de desenvolvimento institucional vigente (CMDI, 2017).

\section{Instrumentos de coleta de dados}

Quanto aos procedimentos de coleta de dados, serão adotados para a fase qualitativa a pesquisa bibliográfica, documental, questionário e entrevista. Esses dois últimos, elaborados para obter a percepção técnica de seus respondentes, com objetivo de coletar as informações da realidade da instituição pesquisada, em aspectos relacionados às compras, às dificuldades logísticas e ao impacto dos custos monetários adicionais de transporte no seu orçamento e nas ações do campus.

As pesquisas bibliográfica e documental fornecerão as informações necessárias para a elaboração do referencial teórico que, na acepção de Oliveira (2011), "servirá de fundamentação para a análise dos dados coletados na realidade pesquisada”. 
Com o propósito de obter respostas significativas que possam estabelecer a realidade das dificuldades externas em um campus interiorano do IFAM, foi elaborado um questionário, que segundo Gil (2019), pode ser definido como "conjunto de questões que são respondidas por escrito pelo pesquisado". As perguntas foram abertas, que conforme Fachin (2001), são aquelas que "dão condição ao pesquisado de discorrer espontaneamente sobre o que se está questionando; as respostas são de livre deliberação, sem limitações e com linguagem própria".

\section{Análise qualitativa}

O presente trabalho se baseou em um questionário como forma de coletar as informações qualitativas, a fim de identificar padrões nas respostas obtidas que melhor pudessem descrever a problemática evidenciada. A pesquisa se desenvolveu considerando seis perguntas, conforme apresentadas a seguir:

1. Como são executadas no IFAM - Campus São Gabriel da Cachoeira, as compras públicas de bens e serviços?

2. A distância geográfica dos fornecedores interfere nas compras da instituição? Em caso positivo, poderia descrever algumas situações?

3. Há diferenciação explicita de preços de bens e serviços entregues na capital do Estado e em São Gabriel da Cachoeira? Em caso positivo, poderia exemplificar alguns casos?

4. Existe um determinado tempo no ano que o processo de entrega dos bens adquiridos e menos oneroso ao campus?

5. Em sua opinião quais medidas ou mudanças deveriam ser adotadas para facilitar a realização das compras? O que precisa ser aperfeiçoado e/ou quais inovações precisam ser introduzidas? O que precisa ser feito para melhorar?

6. Há alguma informação complementar que você gostaria de acrescentar? Algo que considera importante e que não foi mencionado?

O questionário foi direcionado aos participantes através da plataforma Google Forms e uma entrevista, por meio de vídeo conferencia pelo aplicativo Google Meet, foi realizada com o diretor do campus, a fim de garantir o consentimento e clareza dos objetivos da pesquisa, bem como as formas que os resultados serão aplicados. O 
formulário teve como foco principal os profissionais responsáveis pelo departamento de administração e núcleo de compras do Instituto Federal do Amazonas.

Inicialmente, um pré-teste foi realizado com cinco servidores da reitoria de três campi do IFAM localizados na cidade de Manaus e que desempenham tarefas semelhantes ao campus analisado. As respostas desse pré-teste foram analisadas para identificar eventuais falhas na clareza e compreensão das questões apresentadas. Dessa forma, foi possível realizar reformulações no questionário para evitar dúvidas no seu preenchimento antes de seu envio à unidade de pesquisa. Tendo em vista a natureza multicampi do IFAM, o questionário aberto foi enviado por meio eletrônico aos servidores envolvidos no processo de compras da unidade pesquisada. Os participantes responderam aos questionários de forma voluntária, em que foram entrevistados 4 servidores diretamente envolvidos com a temática da pesquisa, além do diretor do instituto.

\section{Fase qualitativa}

$\mathrm{Na}$ fase qualitativa, os dados foram analisados mediante técnica de análise de conteúdo de Bardin (2016), estruturada em três etapas:

1. Pré-análise: seleção de dados e sistematização das ideias da pesquisa;

2. Exploração do material: codificação e categorização dos dados;

3. Tratamento dos resultados, inferência e interpretação: os dados destacados receberam tratamento analítico para que se tornassem significantes e válidos para serem analisados criticamente, visando responder aos objetivos estabelecidos da investigação.

A identificação de padrões foi estruturada com o suporte do software de análise Atlas t.i versão 9. Silva e Leão (2018) destacam que esse programa é um dos mais eficientes em relação as análises qualitativas, principalmente no que se refere ao auxílio na organização de dados e palavras, onde essas podem ser codificadas para que seja possível perceber e identificar os padrões existentes. Nunes et al. (2019) completam que devido às análises desses dados serem em grande parte intuitiva, ou seja, os resultados podem ser interpretados conforme discernimento do pesquisador, a utilização de softwares de análise contribui para o fortalecimento das ideias necessárias, garantindo uma análise consistente sem desvios em relação ao centro da pesquisa. 


\section{Fase quantitativa - estatística descritiva}

Foi realizada uma análise descritiva dos dados referentes aos custos de aquisição dos produtos alimentícios, usando planilhas eletrônicas e os resultados foram apresentados em tabelas de distribuição de frequências e gráficos. Destaca-se que o grupo de aquisição relacionado aos alimentos foi identificado como a principal variável, levando em considerações sua maior rotatividade no campi pesquisado. Através da técnica de estatística descritiva simples, foram obtidos resultados básicos como mínimo, máximo, soma, contagem e média.

Para Toledo e Ovalle (2011), a estatística descritiva busca transformar dados quantitativos obtidos em resultados mais facilmente interpretados, através de gráficos e tabelas, bem como do cálculo de coeficientes, de forma a descrever resumidamente fenômenos observados. Conforme apresentaram Fávero e Belfiore (2017), a estatística descritiva é fundamental para a análise de dados, sendo necessariamente desenvolvida para estudo de informações quantitativas. Por meio dos resultados agrupados e ajustados de acordo com o tipo de informação que se pretende extrair, é possível resumir uma quantidade significativa de informações, destacando precisamente a essência geral dos dados apresentados, reduzindo assim a quantidade de informações.

$\mathrm{Na}$ Tabela 1, apresenta-se as principais funções da estatística descritiva que serão abordadas pela presente pesquisa.

Tabela 1 - Principais funções da estatística descritiva

\begin{tabular}{|c|c|c|}
\hline $\begin{array}{l}\text { Estatística } \\
\text { descritiva }\end{array}$ & Fórmula & Descrição \\
\hline Mínimo & Mínimo $(X: X 1)$ & Identificação do menor valor em um intervalo \\
\hline Máximo & Máximo $(X: X 1)$ & Identificação do maior valor em um intervalo \\
\hline Soma & $\Sigma=(X: X 1)$ & $\begin{array}{c}\text { Somatórios dos valores dos elementos de } \\
\text { determinado intervalo }\end{array}$ \\
\hline Contagem & $\sum=(X: X 1)$ & Contagem dos elementos de determinado intervalo \\
\hline Média & $\bar{X}=\frac{\Sigma X n}{n}$ & $\begin{array}{c}\mathrm{X} \text { representa a média de um determinado intervalo } \\
\text { de valores }\end{array}$ \\
\hline
\end{tabular}

Sampaio et al. (2018) destacaram que as funções de mínimo e máximo representam, respectivamente, o menor e maior valor em relação a um intervalo de dados. A soma apresenta a combinação total dos valores de dados destacados, e neste sentido, a contagem da quantidade de intervalos da amostra. Por outro lado, a média destaca o nível geral dos intervalos analisados. 


\section{RESULTADOS E DISCUSSÃO}

\section{Análise qualitativa}

As análises se concentraram em construir uma nuvem de palavras, onde a intenção da construção é apresentar a frequência com que determinadas palavras são citadas em relação a um conjunto de ideias. Dessa maneira, é possível destacar padrões de escritas que podem ser consideradas similares quando em comparação com todos os envolvidos na pesquisa, conforme os resultados apresentados na Figura 2.

Figura 2 - Nuvem de palavras

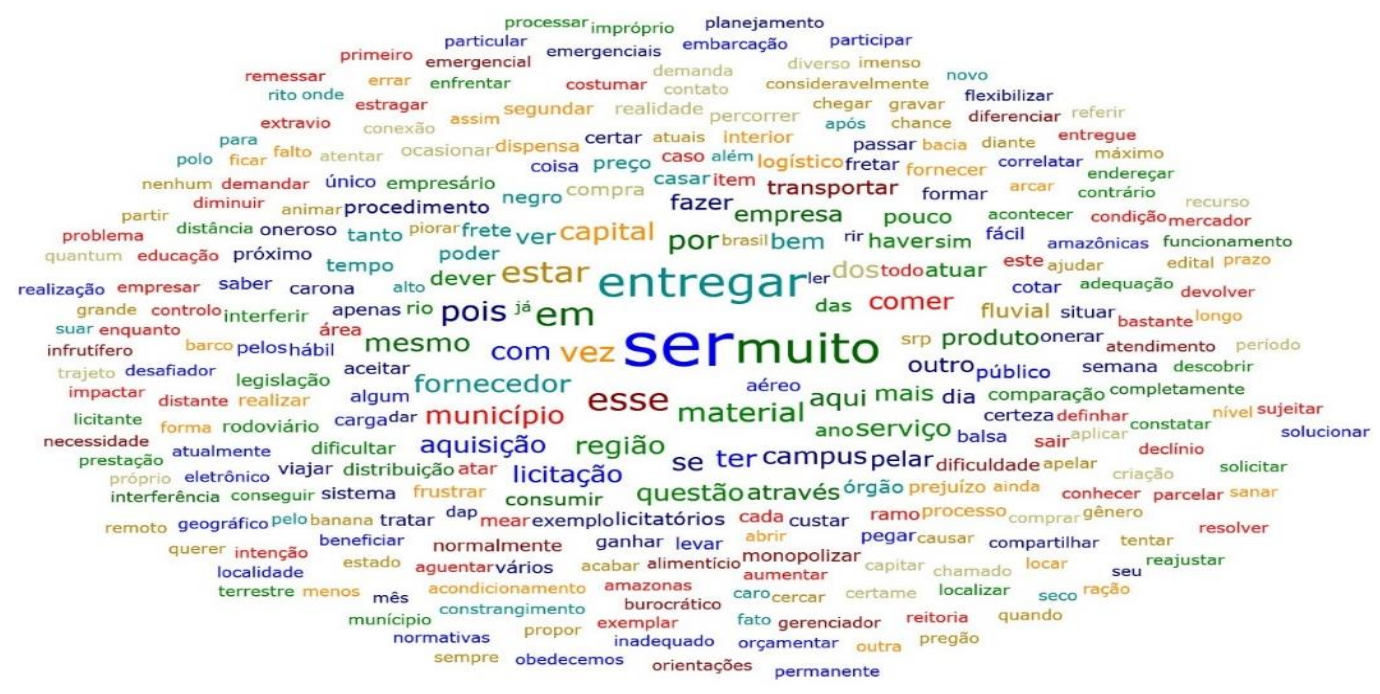

Analisando as informações apresentadas na nuvem de palavras da Figura 2, verifica-se que algumas apresentaram significativos destaques. Como por exemplo, podese destacar a palavra aquisição, que é o foco principal das atividades dos entrevistados que atuam no setor de compras. Associado a essa condição, tem-se o termo licitação. Considerando que se trata de um instituto federal, as compras devem seguir esse tipo de regimento. Contudo, é possível notar outras palavras como: entrega, dificuldade, burocrático, fluvial, logística, oneroso etc. Esses últimos padrões ortográficos indicam condições de riscos que potencialmente podem resultar em constantes prejuízos nas operações destacadas.

Com o objetivo de organizar os resultados obtidos, as respostas foram estruturadas por meio de códigos, onde podem ser considerados padrões de respostas que pretendem transmitir um parâmetro didático. Esses códigos estão representados na Tabela 2. 
Tabela 2 - Códigos identificados

\begin{tabular}{lc}
\hline \multicolumn{1}{c}{ Códigos } & Questões observadas \\
\hline Aumento dos preços anualmente & $4)$ \\
Custos operacionais & Em todas as questões \\
Dificuldades no trajeto & 3 ) \\
Distância & $2)$ \\
Estratégias de licitação e contato & $5)$ \\
Favorecimento na licitação & $2)$ \\
Grande diferenciação de preços & $3)$ \\
Maior atenção e conhecimento da realidade & $6)$ \\
Processo de licitação & $1)$ \\
\hline
\end{tabular}

Os códigos apresentados na Tabela 2 são relativos aos padrões das respostas obtidas pelos entrevistados. Em relação ao código aumento dos preços anualmente, verificou-se que a cada ano os custos da operação são elevados em decorrência, principalmente, do aumento aquisitivo dos materiais, fator que consequentemente exerce um impacto administrativo nos procedimentos de aquisição.

O código custos operacionais estão associados aos códigos apresentados anteriormente. Foi em comum consentimento dos entrevistados que manter os custos é uma tarefa praticamente impossível levando em consideração que existe uma prática comum de aumento de preços dos materiais por parte, principalmente, dos fornecedores.

Dificuldades no trajeto e distância são códigos que representam um fator comum na região amazônica, principalmente nos municípios distantes das capitais, como é o caso de São de Gabriel da Cachoeira. São duas principais condições que podem explicar os dois códigos apresentados anteriormente, pois as empresas responsáveis no atendimento às solicitações de materiais consideram os custos operacionais na composição dos preços.

Em relação ao código estratégias de licitação e contato, verificou-se, principalmente, que existe uma preocupação dos entrevistados nas ações futuras, sendo de grande necessidade que existam melhores planejamento de licitações, onde haja um melhor contato entre as instituições e as possíveis empresas fornecedoras.

Quanto ao código favorecimento na licitação, verificou-se, principalmente, que os custos relativos à aquisição e logística dos materiais até a instituição é bastante complexa, condição que potencialmente levam os entrevistados a acreditarem que as empresas que fazem parte do pregão buscam favorecer principalmente as cidades das quais os envios dos produtos apresentem certa facilidade, não sendo este o caso de São Gabriel da Cachoeira, distante, aproximadamente, $800 \mathrm{~km}$ da capital. 
Por outro lado, no caso da grande diferenciação de preços, verifica-se que o código está relativo aos custos operacionais e dificuldades no trajeto. Nessa etapa, destaca-se que existe uma correlação direta entre os custos da operação, principalmente no que se refere à logística, de modo que se nota diferenciação de valores dos produtos entre cidades que possuem fácil acesso e aquelas com maiores dificuldades no trajeto.

Em relação ao código maior atenção e conhecimento da realidade, refere-se à condição de isolamento do município, onde parte dos pesquisadores destacaram que para que ocorra uma real melhoria nos procedimentos de aquisição dos materiais, faz-se necessário que as empresas envolvidas no processo de licitação devam, efetivamente, conhecer a realidade e dificuldades da região, pois não é incomum casos em que as empresas cancelem os pedidos por não terem o conhecimento prévio das condições de logística que devem atender às solicitações, cujas dificuldades de trajeto via fluvial são significativamente reduzidas quando os rios estão no período de cheia e bastante dificultosas no período de estiagem.

O código relativo ao processo de licitação destaca que todos os entrevistados realizam suas atribuições com base em processos de licitações, onde é necessário determinado tempo para que as operações das aquisições sejam efetivadas, representando períodos longos de abastecimento devido à burocracia envolvida.

Esses resultados evidenciam as adversidades que a instituição enfrenta, de modo que, por conta de sua localização, a distância e dificuldades em relação à logística, resultam em constantes prejuízos aos profissionais e alunos.

\section{Análise quantitativa}

Para esta análise, considerou-se a aquisição de produtos alimentícios entre os anos de 2018 e 2020, onde foram realizadas comparações entre o campus de São Gabriel da Cachoeira (CSGC) e Manaus (CMAO). A Figura 3 apresenta os custos obtidos no intervalo de tempo considerado. 
Figura 3 - Valores gerais de aquisição

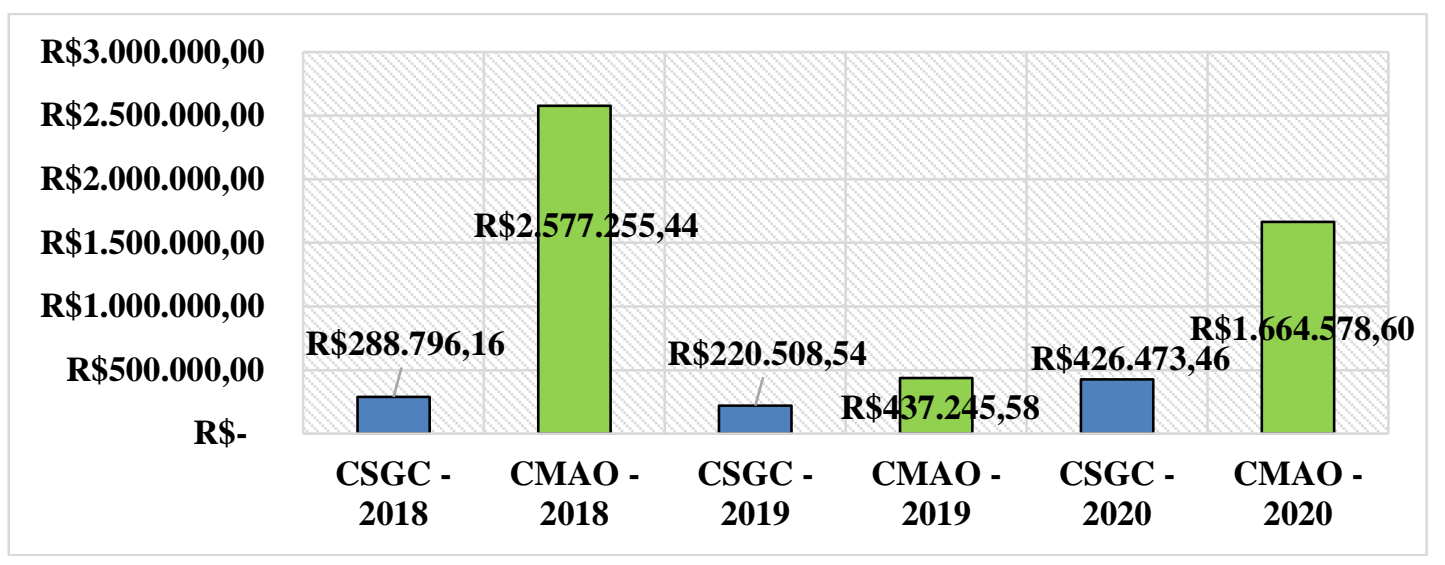

Considerando a quantidade de instituições, alunos e servidores, os custos de aquisição dos produtos no órgão da capital apresentam valores significativamente mais elevados, principalmente no ano de 2018 , obtendo um custo total superior a $\mathrm{R} \$ 2,5$ milhões. Destaca-se também os custos referentes a 2020, que mesmo considerando a paralisação de algumas atividades por conta da pandemia da COVID-19, os custos de aquisição foram superiores a R \$ 1,6 milhões. Por outro lado, considerando o mesmo período para o CSGC, verifica-se que as diferenças totais dos custos também apresentaram significativas alterações de valores, principalmente quando comparado os anos de 2019 e 2020 . Condição que pode ser explicada devido à quantidade total de itens adquiridos, mas principalmente ao aumento dos preços praticados pelos fornecedores.

Para uma melhor visualização desses valores, incialmente, organizou-se os itens adquiridos em categorias, sendo classificadas como: A; B; C; D; E; F; G; H; I; J; e L. Destaca-se que todas as categorias são correspondentes ao gênero alimentício, em que as informações de valor relacionadas a cada categoria se referem a média dos produtos equivalentes e que integram as mesmas, sendo esse considerado o de maior rotatividade nos campi, organizados conforme descrito na Tabela 3. 
Tabela 3 - Aquisições em 2018

\begin{tabular}{|c|c|c|c|c|c|c|c|}
\hline \multirow[b]{2}{*}{ Produto } & \multicolumn{3}{|c|}{ CSGC } & \multicolumn{3}{|c|}{ CMAO } & \multirow{2}{*}{$\begin{array}{c}\text { Aumento/ } \\
\text { redução } \\
(\%)\end{array}$} \\
\hline & Und. & $\begin{array}{l}\text { Valor } \\
\text { (Und.) } \\
\end{array}$ & Total & Und. & $\begin{array}{l}\text { Valor } \\
\text { (Und.) }\end{array}$ & Total & \\
\hline A & 9721 & $\mathrm{R} \$ 12,30$ & $\mathrm{R} \$ 119.568,30$ & 71183 & $\mathrm{R} \$ 12,29$ & $\mathrm{R} \$ 874.839,07$ & 0,08 \\
\hline B & 467 & $\mathrm{R} \$ 16,30$ & $\mathrm{R} \$ 7.612,10$ & 13500 & $\mathrm{R} \$ 13,11$ & $\mathrm{R} \$ 176.985,00$ & 24,33 \\
\hline $\mathrm{C}$ & 5605 & $\mathrm{R} \$ 7,07$ & $\mathrm{R} \$ 39.627,35$ & 77000 & $\mathrm{R} \$ 3,00$ & $\mathrm{R} \$ 231.000,00$ & 135,67 \\
\hline $\mathrm{D}$ & 10485 & $\mathrm{R} \$ 5,79$ & $\mathrm{R} \$ 60.708,15$ & 73500 & $\mathrm{R} \$ 4,16$ & $\mathrm{R} \$ 305.760,00$ & 39,18 \\
\hline $\mathrm{E}$ & 9058 & $\mathrm{R} \$ 6,24$ & $\mathrm{R} \$ 56.521,92$ & 86370 & $\mathrm{R} \$ 7,12$ & $\mathrm{R} \$ 614.954,40$ & $-12,36$ \\
\hline $\mathrm{F}$ & 5200 & $\mathrm{R} \$ 3,85$ & $\mathrm{R} \$ 20.020,00$ & 43200 & $\mathrm{R} \$ 4,34$ & $\mathrm{R} \$ 187.488,00$ & $-11,29$ \\
\hline G & 1127 & $\mathrm{R} \$ 9,35$ & $\mathrm{R} \$ 10.537,45$ & 10100 & $\mathrm{R} \$ 6,38$ & $\mathrm{R} \$ 64.438,00$ & 46,55 \\
\hline $\mathrm{H}$ & 2858 & $\mathrm{R} \$ 5,22$ & $\mathrm{R} \$ 14.918,76$ & 54307 & $\mathrm{R} \$ 3,57$ & $\mathrm{R} \$ 193.875,99$ & 46,22 \\
\hline I & 5129 & $\mathrm{R} \$ 1,89$ & $\mathrm{R} \$ 9.693,81$ & 4224 & $\mathrm{R} \$ 2,49$ & $\mathrm{R} \$ 10.517,76$ & $-24,10$ \\
\hline $\mathrm{J}$ & 2552 & $\mathrm{R} \$ 2,88$ & $\mathrm{R} \$ 7.349,76$ & 22000 & $\mathrm{R} \$ 3,38$ & $\mathrm{R} \$ 74.360,00$ & $-14,79$ \\
\hline $\mathrm{L}$ & 995 & $\mathrm{R} \$ 6,86$ & $\mathrm{R} \$ 6.825,70$ & 23200 & $\mathrm{R} \$ 4,90$ & $\mathrm{R} \$ 113.680,00$ & 40,00 \\
\hline Total & 53197 & $\mathbf{R} \$ 77,75$ & $\mathbf{R} \$ 353.383,30$ & 478584 & R\$59,84 & $\mathrm{R} \$ 2.847 .898,22$ & 29,93 \\
\hline
\end{tabular}

Os valores apresentados nas Tabela 3 foram obtidos por meio de vários fornecedores, pois todos os processos de aquisição de materiais e serviços por meio de licitação são abertos para todo território brasileiro. Como o produto analisado é alimentício, alguns fornecedores de produtos são específicos, mas sempre usando a instrução da lei em que o ganhador é o que oferta o preço mais vantajoso, conforme demanda e necessidades dos campi analisados.

No ano de 2018, os campi adquiriram diferentes quantidades de produtos. Portanto, a fim de permitir a comparação dos gastos entre as instituições, os valores foram organizados em termos dos preços unitários. Na coluna aumento/redução (\%), o cálculo foi realizado para comparar o aumento ou a redução entre os dois campi, utilizando como base o valor do CMAO. Nesse sentido, os preços para os produtos E, F, I e J apresentaram reduções para o CSGC em comparação ao CMAO. Contudo, outros produtos como o C, apresentou um aumento para o CSGC que foi de 135,67\% do valor do CMAO. Além disso, pode-se verificar que os produtos D, G e H apresentaram aumentos para o CSGC de $39 \%$ a $47 \%$ em comparação com o CMAO, elevando os custos de aquisição. Em termos dos preços unitários, verifica-se que os produtos do CSGC apresentam aumentos de 29,93\% em relação ao CMAO no ano de 2018.

$\mathrm{Na}$ Figura 4 é apresentado um gráfico para analisar o quantitativo referente aos custos de aquisição, bem como a diferença para os dois campi. 
Figura 4 - Comparativo de dados 2018

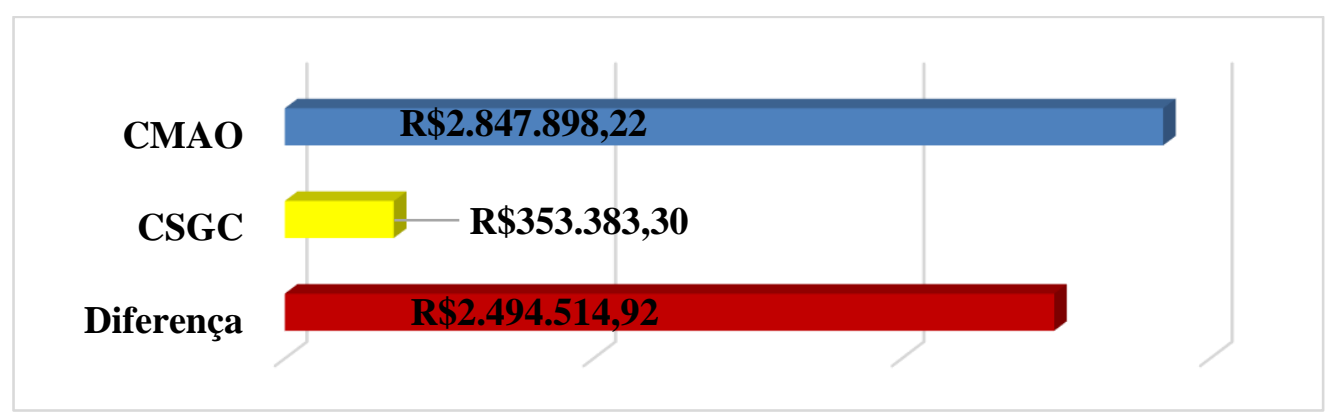

A diferença de valores apresentados na Figura 4 é equivalente ao valor total dos insumos alimentícios adquiridos pelo CMAO. A barra denominada de diferença corresponde a subtração dos custos do CMAO em relação ao CSGC. Nesse caso, verificase que em 2018 o CMAO obteve um custo de aquisição unitário superior a 29\%, quando em comparação com CSGC, indicando que a quantidade dos produtos adquiridos teve impacto significativo no total dos custos. A Tabela 4 apresenta as aquisições no ano de 2019.

Tabela 4 - Aquisições em 2019

\begin{tabular}{|c|c|c|c|c|c|c|c|}
\hline \multirow[b]{2}{*}{$\begin{array}{l}\text { Produt } \\
\quad \text { o }\end{array}$} & \multicolumn{3}{|c|}{ CSGC } & \multicolumn{3}{|c|}{ CMAO } & \multirow{2}{*}{$\begin{array}{c}\text { Aumento } \\
\text { /redução } \\
(\%)\end{array}$} \\
\hline & Und. & $\begin{array}{l}\text { Valor } \\
\text { (Und.) }\end{array}$ & Total & Und. & $\begin{array}{l}\text { Valor } \\
\text { (Und.) }\end{array}$ & Total & \\
\hline A & 3020 & $\mathrm{R} \$ 16,20$ & $\mathrm{R} \$ 48.924,00$ & 8250 & $\mathrm{R} \$ 11,66$ & $\mathrm{R} \$ 96.195,00$ & 38,94 \\
\hline B & 384 & $\mathrm{R} \$ 13,50$ & $\mathrm{R} \$ 5.184,00$ & 1400 & $\mathrm{R} \$ 3,34$ & $R \$ 4.676,00$ & 304,19 \\
\hline $\mathrm{C}$ & 2212 & $\mathrm{R} \$ 8,82$ & $\mathrm{R} \$ 19.509,84$ & 4000 & $\mathrm{R} \$ 3,73$ & $\mathrm{R} \$ 14.920,00$ & 136,46 \\
\hline $\mathrm{D}$ & 8263 & $\mathrm{R} \$ 10,09$ & $\mathrm{R} \$ 83.373,67$ & 8691 & $\mathrm{R} \$ 7,65$ & $\mathrm{R} \$ 66.486,15$ & 31,90 \\
\hline $\mathrm{E}$ & 5360 & $\mathrm{R} \$ 7,73$ & $\mathrm{R} \$ 41.432,80$ & 8600 & $\mathrm{R} \$ 6,52$ & $\mathrm{R} \$ 56.072,00$ & 18,56 \\
\hline $\mathrm{F}$ & 3618 & $\mathrm{R} \$ 4,78$ & $\mathrm{R} \$ 17.294,04$ & 29960 & $\mathrm{R} \$ 4,18$ & $\mathrm{R} \$ 125.232,80$ & 14,35 \\
\hline G & 802 & $\mathrm{R} \$ 13,83$ & $\mathrm{R} \$ 11.091,66$ & 300 & $\mathrm{R} \$ 6,22$ & $\mathrm{R} \$ 1.866,00$ & 122,35 \\
\hline $\mathrm{H}$ & 1281 & $\mathrm{R} \$ 7,35$ & $\mathrm{R} \$ 9.415,35$ & 17090 & $\mathrm{R} \$ 4,27$ & $\mathrm{R} \$ 72.974,30$ & 72,13 \\
\hline I & 1273 & $\mathrm{R} \$ 9,67$ & $\mathrm{R} \$ 12.309,91$ & 4900 & $\mathrm{R} \$ 2,48$ & $\mathrm{R} \$ 12.152,00$ & 289,92 \\
\hline $\mathrm{J}$ & 1275 & $\mathrm{R} \$ 2,98$ & $\mathrm{R} \$ 3.799,50$ & 11020 & $\mathrm{R} \$ 3,03$ & $\mathrm{R} \$ 33.390,60$ & $-1,65$ \\
\hline $\mathrm{L}$ & 574 & $\mathrm{R} \$ 9,00$ & $\mathrm{R} \$ 5.166,00$ & 1700 & $\mathrm{R} \$ 6,05$ & $\mathrm{R} \$ 10.285,00$ & 48,76 \\
\hline Total & 53197 & 103,95 & $257.500,77$ & 478584 & 59,13 & $494.249,85$ & 75,80 \\
\hline
\end{tabular}

Semelhante às condições observadas no ano de 2018, alguns produtos tiveram aumentos significativos em comparação aos campi analisados, onde além do fator logística, os custos operacionais ocorreram devido aos ajustes anuais dos valores praticados. Somente os custos de aquisição para o produto J é menor para o CSGC. Por outro lado, destaca-se os produtos B e I que foram superiores para o CSGC em comparação ao CMAO. Além desses, os produtos C e $\mathrm{G}$ também apresentaram um aumento para o CSGC que foi de $136,46 \%$ e $122,35 \%$, respectivamente, dos valores do 
CMAO. Em geral, verificou-se um aumento de 75,80\% para o CSGC, utilizando como base os produtos do CMAO.

Na Figura 5 é apresentado um gráfico do quantitativo referente aos custos de aquisição, bem como a diferença para os dois campi.

Figura 5 - Comparativo de dados 2019

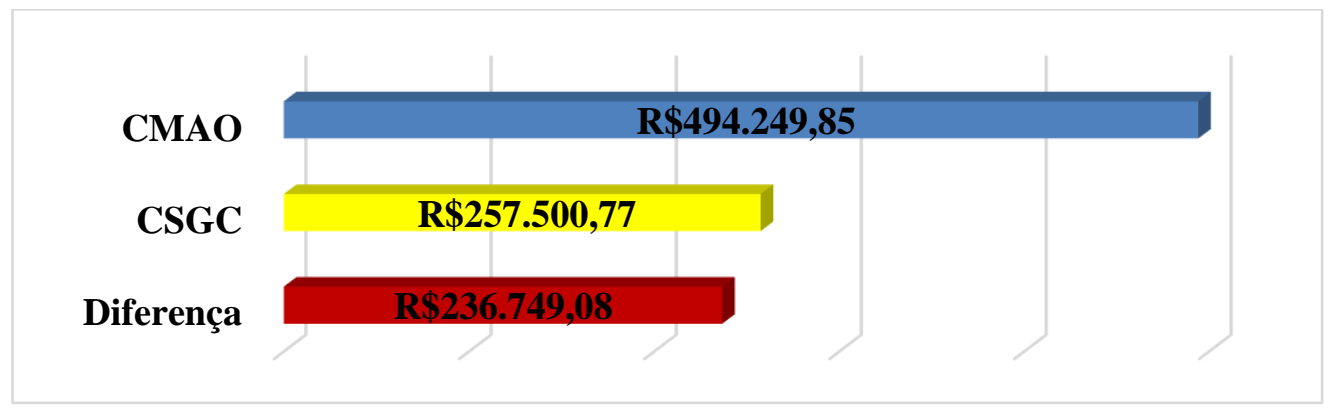

Conforme apresentado na Figura 5, os investimentos em produtos alimentícios possuem diferença significativa entre os campi analisados, sendo de R $236.749,08$. Destaca-se que esse valor é de aproximadamente 75\% maior para o CSGC. Além disso, nota-se que ao contrário dos percentuais de diferenças apresentados no ano de 2018, houve um aumento substancial dos valores para o CSGC. A Tabela 5 apresenta as informações de aquisição, considerando as categorias relativas ao ano de 2020.

Tabela 5 - Aquisições em 2020

\begin{tabular}{|c|c|c|c|c|c|c|c|}
\hline \multirow[b]{2}{*}{$\begin{array}{c}\text { Produt } \\
\quad 0 \\
\end{array}$} & \multicolumn{3}{|c|}{ CSGC } & \multicolumn{3}{|c|}{ CMAO } & \multirow{2}{*}{$\begin{array}{c}\text { Aument } \\
\text { o/reduçã } \\
\text { o }(\%)\end{array}$} \\
\hline & Und. & $\begin{array}{l}\text { Valor } \\
\text { (Und.) }\end{array}$ & Total & Und. & $\begin{array}{l}\text { Valor } \\
\text { (Und.) }\end{array}$ & Total & \\
\hline A & 3 & $\mathrm{R}$ & $\mathrm{R}$ & 58206 & $\begin{array}{c}\mathrm{R} \$ 11,6 \\
7\end{array}$ & 2 & 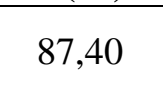 \\
\hline B & & & & & $\mathrm{R} \$ 6,00$ & & \\
\hline $\mathrm{C}$ & 1799 & & & & & & \\
\hline $\mathrm{D}$ & 13387 & & $\mathrm{R} \$ 2$ & & & & 171,56 \\
\hline $\mathrm{E}$ & 10224 & $\mathrm{R} \$ 12,18$ & $\mathrm{R} \$ 1$ & & 34 & & \\
\hline $\mathrm{F}$ & 3603 & & & 44000 & 81 & $\mathrm{R} \$$ & \\
\hline G & 1295 & $\mathrm{R} \$ 12,10$ & & & 05 & & 00 \\
\hline $\mathrm{H}$ & 4370 & $\mathrm{R} \$ 6,73$ & $\mathrm{R} \$ 29$ & 36702 & $\mathrm{R} \$ 3,17$ & $\mathrm{R} \$ 116.3$ & 112,30 \\
\hline I & 3547 & $\mathrm{R} \$ 2,70$ & & 4440 & $\mathrm{R} \$ 2,95$ & $\mathrm{R} \$ 13.098,00$ & $-8,47$ \\
\hline $\mathrm{J}$ & 2324 & $\mathrm{R} \$ 4,31$ & $\mathrm{R} \$ 10.016,44$ & 441 & $\mathrm{R} \$ 1,85$ & $\mathrm{R} \$ 815,85$ & 132,97 \\
\hline $\mathrm{L}$ & 522 & $\mathrm{R} \$ 12,17$ & $\mathrm{R} \$ 6.352,74$ & 15350 & $\mathrm{R} \$ 6,38$ & $\mathrm{R} \$ 97.933,00$ & $90,75 \%$ \\
\hline Total & 44897 & 120,90 & 519.988,79 & 344989 & 56,80 & 2.003.744,21 & 112,85 \\
\hline
\end{tabular}

Verifica-se na Tabela 5 que, apesar das quantidades serem menores em relação ao ano de 2018, os valores unitários e, consequentemente o total, são maiores. Condição 
evidenciada na análise qualitativa quanto aos aumentos de custos maiores para a unidade do CSGC em comparação com o CMAO, onde em relação a esse levantamento, somente os custos de aquisição para o produto I é menor para o CSGC. Os valores das categorias B, C e D apresentaram aumentos de $175 \%, 454,77 \%$ e 171,56\%, respectivamente, para o CSGCem comparação com o CMAO Os custos de aquisição para o CSGC apresentaram um aumento de $112,85 \%$ quando comparado com o CMAO.

$\mathrm{Na}$ Figura 6 é apresentado um gráfico do quantitativo referente aos custos de aquisição, bem como a diferença para os dois campi.

Figura 6 - Comparativo de dados 2020

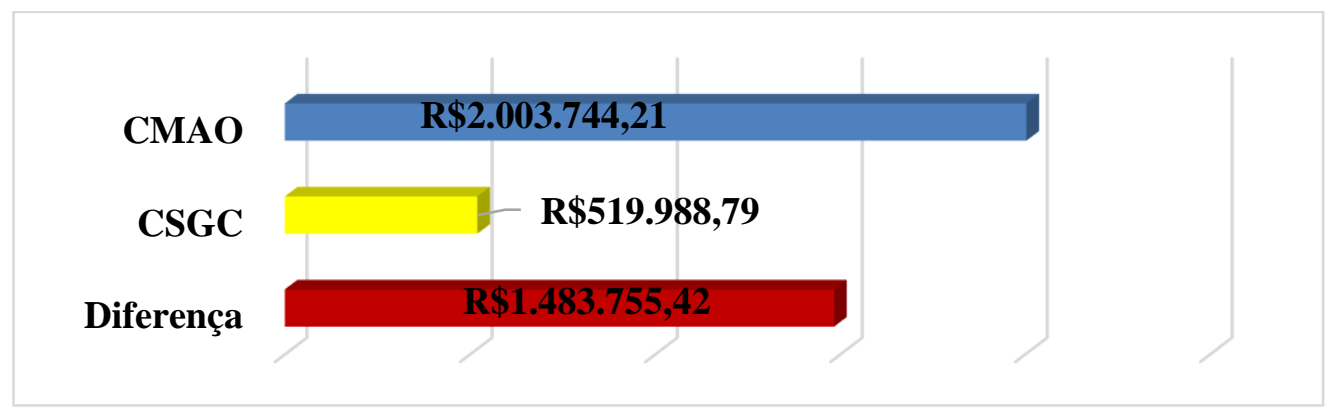

Em análise aos dados de aquisição referente ao ano de 2020, nota-se que a diferença total de valores nos campis analisados é consideravelmente maior em relação aos anos anteriores, atingindo o valor de $\mathrm{R} \$ 1.483 .755,42$.

\section{Análise quantitativa: estatística descritiva}

A Tabela 6 apresenta os resultados gerais da aplicação da estatística descritiva para os dois campi, considerando os anos de 2018 a 2020.

Tabela 6 - Análise descritiva

\begin{tabular}{|c|c|c|c|c|c|c|}
\hline \multirow{2}{*}{ Análise } & \multicolumn{3}{|c|}{ CSGC } & \multicolumn{3}{|c|}{ CMAO } \\
\hline & 2018 & 2019 & 2020 & 2018 & 2019 & 2020 \\
\hline Contagem & 56 & 56 & 56 & 56 & 56 & 56 \\
\hline Mínimo & $\mathrm{R} \$ 1,22$ & $\mathrm{R} \$ 1,65$ & $\mathrm{R} \$ 2,03$ & $\mathrm{R} \$ 0,77$ & $\mathrm{R} \$ 1,37$ & $\mathrm{R} \$ 1,20$ \\
\hline Máximo & $\mathrm{R} \$ 21,15$ & $\mathrm{R} \$ 33,00$ & $\mathrm{R} \$ 42,00$ & $\mathrm{R} \$ 28,72$ & $\mathrm{R} \$ 22,76$ & $\mathrm{R} \$ 26,66$ \\
\hline Soma & $\mathrm{R} \$ 77,75$ & $\mathrm{R} \$ 103,95$ & $\mathrm{R} \$ 120,88$ & $\mathrm{R} \$ 64,74$ & $\mathrm{R} \$ 59,13$ & $\mathrm{R} \$ 56,80$ \\
\hline Média & $\mathrm{R} \$ 7,07$ & $\mathrm{R} \$ 9,45$ & $\mathrm{R} \$ 10,99$ & $\mathrm{R} \$ 5,89$ & $\mathrm{R} \$ 5,38$ & $\mathrm{R} \$ 5,16$ \\
\hline
\end{tabular}


A análise estatística descritiva apresentada na Tabela 6 indica as cinco principais informações desse tipo de análise, sendo essas: contagem, que indica a quantidade de itens analisados, sendo 56, que são os itens de maior rotatividade para todos os intervalos de anos destacados. As observações de mínimo e máximo que representam, respectivamente, os menores e maiores custos dos produtos adquiridos.

Por outro lado, o resultado relativo à soma representa o montante dos valores unitários de cada produto adquirido nos intervalos analisados. E com base nessas informações, destaca-se a média, sendo considerada como um valor intermediário em relação ao preço dos itens adquiridos.

Com base nos resultados obtidos, evidencia-se que, considerando o CSGC, ocorreu um aumento significativo dos preços, onde a compra total para o ano de 2020 apresentou um percentual equivalente a 55,47\% maior quando comparado com 2018. Esse aumento não está associado com a quantidade de insumos adquiridos, mas sim com os custos operacionais que seguem uma tendência de aumento a cada ano efetivo.

Contudo, em análise as informações do CMAO, nota-se que os valores totais apresentaram uma redução, cujo valor em 2020 foi 12,26\% menor quando comparado com 2018, sugerindo que nesse caso os custos operacionais não afetaram de maneira significativa a aquisição dos insumos, podendo dessa maneira haver oscilações de valores entre os períodos de compras.

\section{Análise comparativa de dados}

Considerando a logística como a principal condição crítica das atividades comerciais da região amazônica, nota-se que essa condição está potencialmente envolvida nas análises qualitativas e quantitativas. Na mesma proporção de sua extensão territorial, o estado do Amazonas apresenta graves problemas em disponibilidade de sistemas logísticos que possam atender as demandas nos 62 municípios, incluindo a capital, que também sofre com algumas adversidades no que se refere à disposição e aquisição de insumos.

Analisando essa realidade e comparando com os resultados obtidos por meio das análises qualitativa e quantitativa, é possível notar maiores índices e criticidade quando o município fica consideravelmente distante da capital Manaus. Considerando as informações apresentadas na pesquisa, nota-se que a distância e a dificuldade de logística 
da cidade de São Gabriel da Cachoeira são as principais preocupações no abastecimento de materiais, sendo necessários custos adicionais para manter as operações no instituto objeto de pesquisa deste trabalho.

$\mathrm{Na}$ análise qualitativa, os entrevistados relataram que a dificuldade operacional, devido à distância do município, resulta em significativos prejuízos institucionais, principalmente no recebimento de insumos necessários para manter os procedimentos administrativos da instituição. Existe uma grande escassez de fornecedores que se dispõem a garantir o abastecimento em locais considerados isolados das grandes cidades, resultando em preços maiores.

Considerando Manaus como modelo comparativo, evidencia-se que alguns fatores como: quantidade solicitada e fenômenos dos rios, como cheias e vazantes, implicam diretamente nos valores unitários praticados. Considerando as seguintes condições: quantidades de aquisição dos produtos e cheias, obtém-se preços baixos devido à relativa facilidade no transporte dos insumos. Por outro lado, quantidades de produtos a serem adquiridos e vazantes, indicam valores relativamente maiores devido às dificuldades no trajeto em função da seca dos rios.

Contudo, destaca-se que mesmo no período das cheias dos rios, onde existe uma certa facilidade no transporte de insumos a municípios que não possuem ligações terrestres, não é possível perceber as mesmas práticas de preços observados em Manaus, quando refere-se a São Gabriel da Cachoeira. Sendo assim, independente das condições, os custos necessários para aquisição de materiais são maiores que na capital.

Essas condições ficam evidentes e perceptíveis no decorrer da análise quantitativa através da identificação dos preços unitários de custos para os campi de Manaus e São Gabriel da Cachoeira. Além disso, confirmou-se uma condição verificada ao longo da análise qualitativa que, independentemente da quantidade solicitada, os materiais requisitados pelo CSGC apresentam preços mais altos pelos fornecedores.

Além disso, os resultados da estatística descritiva indicaram que entre os anos analisados, houve um acréscimo significativo de custos direcionadas às aquisições de materiais. Cenário bastante diferente ao que ocorre no CMAO, onde a lei da oferta e demanda se faz efetiva, principalmente considerando a grande quantidade de potenciais fornecedores disponíveis.

Em valores percentuais, em 2018, o CSGC teve um aumento de 29,93\% em comparação com o CMAO; já em 2019, esse percentual foi de 75,80\%, mesmo 
considerando que neste período houve uma redução da quantidade de itens adquiridos. Enquanto que em 2020, registrou-se outro valor significativamente elevado, atingindo um aumento de $112,85 \%$.

\section{CONCLUSÕES}

Os resultados obtidos nesta pesquisa mostraram que a manutenção de algumas atividades nos municípios analisados são um grande desafio principalmente aos gestores e responsáveis, levando em consideração as dificuldades relacionadas à logística. Percepções dessa natureza são identificadas nas mais variadas atividades existentes, onde em condições específicas esse fator tende a desenvolver dificuldades nas operações, resultados em agravantes que, inclusive, podem comprometer o projeto construído.

Nota-se pelas informações verificadas na pesquisa que os fatores logísticos, escassez de fornecedores e custos operacionais são as principais condições adversas que afetam diretamente o processo de aquisição de insumos. Devido à essa condição, não é incomum que haja uma constante falta de materiais críticos na unidade da instituição de São Gabriel da Cachoeira, afetando diretamente os principais objetivos institucionais.

Os custos necessários para manter as operações são significativamente maiores em relação a unidade localizada em Manaus, impossibilitando que ocorram investimentos em áreas específicas, como por exemplo em tecnologias na área da informação, comprometendo os procedimentos educacionais.

Com relação a análise quantitativa, os custos de aquisição passaram por significativos ajustes ao longo do período analisado, em que os custos indicaram percentuais elevados para o CSGC, mesmo considerando quantidade maior de itens adquiridos, condição que pode ser explicada por conta dos reajustes realizados pelos fornecedores.

Considerando os resultados apresentados pela análise quantitativa, faz-se necessário a construção de estratégias que visem a redução dos preços praticados pelos fornecedores, pois, independente do período amazônico em relação ao transporte, ou seja, vazante ou cheia dos rios, os valores seguem uma crescente, não apresentando vantagens competitivas entre os fornecedores, e ainda deixando os custos operacionais do campus mais expressivos. 


\section{AGRADECIMENTOS}

Ao Instituto Federal de Educação, Ciência e Tecnologia do Amazonas/Campus Manaus Distrito Industrial e Campus São Gabriel da Cachoeira.

\section{REFERÊNCIAS}

BARDIN, LAURENCE. Análise de conteúdo. São Paulo: Edições 70/Almedina Brasil, 2016.

CMDI. CAMPUS MANAUS DISTRITO INDUSTRIAL. Esclarecimentos orçamento IFAM-CMDI 2017. Manaus: 2017. Disponível em:

$<$ http://www2.Ifam.edu.br/campus/cmdi/arquivos/esclarecimentos-orcamento-Ifamcmdi-2017.pdf >. Acesso em: 28 de fevereiro de 2020.

COMISSÃO DE DESENVOLVIMENTO URBANO. Projeto de Lei no 3.093, de 2015. Altera a Lei $n^{\circ} 11.977$, de 7 de julho de 2009, que dispõe sobre o Programa Minha Casa, Minha Vida PMCMV), entre outros assuntos, para tratar da incorporação de custos diferenciados para empreendimentos na Amazônia Legal. Disponível em: https://www.camara.leg.br/proposicoesWeb/prop_mostrarintegra?codteor=1389861. Acesso em: 25 de fevereiro de 2020.

FACHIN, ODÍLIA. Fundamentos de Metodologia. São Paulo: Saraiva, 2001.

FÁVERO, L. P., BELFIORE, P. Manual de Análise de Dados: Estatística e Modelagem. Rio de Janeiro: Elsevier, 2017.

GIL, A. C. Como elaborar projetos de pesquisa. 6. ed. São Paulo: Atlas, 2019.

IFAM. INSTITUTO FEDERAL DE EDUCAÇÃO, CIÊNCIA E TECNOLOGIA DO AMAZONAS. IFAM, UFAM e UEA assinam acordo de cooperação. Manaus, AM., 2018. Disponível em <http://www2.ifam.edu.br/noticias/ifam-ufam-e-uea-assinamacordo-de-cooperacao-1>. Acesso em: 10 de fevereiro de 2020.

NUNES, J. V., WOLOSZYN, M., GONÇALVES, B.S., PINTO, M. D. A pesquisa qualitativa apoiada por softwares de análise de dados: uma investigação a partir de exemplos. Revista Fronteiras, v. 19, n. 2, pp. 233-244, 2017.

OLIVEIRA, MARIA MARLY DE. Como fazer projetos, relatórios, monografias, dissertações e teses. 5 ed. Rio de Janeiro: Elsevier, 2011.

PLATAFORMA BRASIL. Brasília, 2020. Disponível em <https://plataformabrasil.saude.gov.br/ login.jsf>. Acesso em: 13 de setembro de 2020. 
SAMPAIO, NILO A., LEONI ROBERTO CAMPOS. Utilização dos testes de hipóteses para média na tomada de decisão. 2015. Disponível em <https://www.aedb.br/wp-content/uploads/2015/05/545.pdf >. Acesso em: 17 de março de 2020.

SILVA, ALLAN GUSTAVO F, et al. A relação entre estado e políticas públicas: uma análise teórica sobre o caso brasileiro. Revista Debates, v. 11, n. 1, pp. 25-42, 2017. Disponível em: <https://seer.ufrgs.br/index.php/debates/article/viewFile/72132/41081>. Acesso em: 7 de outubro de 2019.

SILVA, MARIA DO SOCORRO ROCHA. Bacia hidrográfica do Rio Amazonas: contribuição para o enquadramento e preservação. 2013. 199f. Tese (Doutorado) Programa de Pós Graduação em Química da Universidade Federal do Amazonas, Manaus, 2012.

SOUZA, MARIA IRENE P. DE OLIVEIRA; ZANETTI, ROSANGELA R. Olhares sobre a formação de professores: relatos, reflexões e proposições. Londrina: UEL, 2016.

TOLEDO, GERALDO LUCIANO; OVALLE, IVO IZIDORO. Estatística Básica. 2. ed. São Paulo: Atlas, 2011.

VERGARA, SYLVIA CONSTANT. Projetos e relatórios de pesquisa em administração. 16. ed. São Paulo: Editora Atlas, 2016.

Recebido em: 01/12/2021

Aprovado em: 23/12/2021

Publicado em: 28/12/2021 